\title{
ЧРЕСКОЖНАЯ ЭНДОСКОПИЧЕСКАЯ ГАСТРОСТОМИЯ С УЗ-НАВИГАЦИЕЙ, КАК МЕТОД ВЫБОРА ДЛЯ ОБЕСПЕЧЕНИЯ ЭНТЕРАЛЬНОГО ПИТАНИЯ У ПАЦИЕНТОВ В ХРОНИЧЕСКОМ КРИТИЧЕСКОМ СОСТОЯНИИ
}

\author{
Яковлева А.В., Шайбак А.А., Скворцов А.Е., Яковлев А.А., \\ Крылов К.Ю., Петрова М.В., Щелкунова И.Г., Гречко А.В.
}

Цель. Анализ эффективности УЗ-навигаџии при чрескожной эндоскопической гастростомии для повышения безопасности метода обеспечения пути доставки энтерального питания.

Материалы и методы. Чрескожная эндоскопическая гастростомия методикой «на себя» (риіl), дополненная УЗ навигацчией, была выполнена 115 пациентам с последствиями тяжелой черепно-мозговой травмь (ЧМТ) и иееребральньми инсультами, находящимся в длительном (хроническом) критическом состоянии и имеющим стойкую дисфагию в период их реабилитации в ФНКЦРР.

Результаты. Все пациенты перенесли операцию удовлетворительно и уже через сутки могли получать энтеральное питание. У двух пациентов $(1,7 \%)$ наблюдалось осложнение в виде пневмоперитонеума, которое потребовало дополнительного назначения антибактериальной терапии и благополучно разрешилось через 7-10 дней, без дополнительных хирургических вмешательств. Добавление в методику УЗИ области, определенной по диафаноскопии, оправдало себя в 15-ти случаях (13,0\%): из них с частотой 73,0\% в определенной для пункции точки выявлен край печени, и с частотой 27,0\% выявлена поперечно-ободочная кишка.

Выводы. 1. Чрескожная эндоскопическая гастростомия по методике «риіl» с добавлением УЗ-навигаџии является адекватным способом обеспечения пути энтерального питания у паџиентов в длительном (хроническом) критическом состоянии.

2. Проведение УЗ-навигации области, определенной диафаноскопией, непосредственно перед пункиией желудка, особенно у пациентов с гепатомегалией, избыточной массой тела и у пацчентов, имеющчих в анамнезе 
оперативные вмешательства на брюшной полости, позволило избежать послеоперачионных тяжельх осложнений в 13,0\% случаев, что доказывает ичелесообразность применения исследования, как часть чрескожной эндоскопической гастростомии.

3. Выполняя чрескожную эндоскопическую гастростомию под УЗ-навигаиией, удалось снизить число осложнений.

Ключевые слова: гастростомия; чрескожная эндоскопическая гастростомия; дисфагия; последствия повреждений головного мозга; УЗИ.

\title{
PERCUTANEOUS ENDOSCOPIC GASTROSTOMY WITH ULTRASONIC NAVIGATION AS A METHOD OF CHOICE TO PROVIDE ENTERAL NUTRITION IN PATIENTS WITH CHRONIC CRITICAL CONDITION
}

\author{
Yakovleva A.V., Shaybak A.A., Skvortsov A.E., Yakovlev A.A., \\ Krylov K.Yu., Petrova M.V., Shchelkunova I.G., Grechko A.V.
}

Background. Analysis of the effectiveness of ULTRASOUND navigation in case of percutaneous endoscopic gastrostomy to improve the security method of ensuring the delivery of enteral nutrition.

Materials and methods. Percutaneous endoscopic gastrostomy with the technique "pull», supplemented by ULTRASOUND navigation, was performed in 115 patients with the consequences of severe craniocerebral trauma and cerebral strokes, who are in a chronic critical condition and have persistent dysphagia during their rehabilitation.

Results. All patients underwent surgery satisfactorily and in a day could receive enteral nutrition. In two patients (1.7\%) there was a complication in the form of pneumoperitoneum, which required additional antibacterial therapy and was successfully resolved after 7-10 days, without additional surgical interventions. Adding to the ultrasound technique the area defined by diaphanoscope, proved to be effective in 15 cases $(13,0 \%)$ of them with a frequency of $73,0 \%$ in particular for puncturing points of the detected liver edge, and a rate of $27,0 \%$ revealed transverse colon.

Conclusion. 1. Percutaneous endoscopic gastrostomy with the technique "pull», supplemented by ULTRASOUND navigation, is an adequate way to 
ensure the path of enteral nutrition in patients in long-term (chronic) critical condition.

2. Carrying out the ULTRASOUND navigation of the area determined by diafanoscopy immediately before the puncture of the stomach, especially in patients with hepatomegaly, overweight and in patients with a history of surgical interventions in the abdominal cavity, allowed to avoid postoperative severe complications in $13 \%$ of cases, which proves the feasibility of the study, as part of percutaneous endoscopic gastrostomy.

3. Performing the percutaneous endoscopic gastrostomy under the ULTRASOUND navigation, it was possible to reduce the number of complications.

Keywords: gastrostomy; percutaneous endoscopic gastrostomy; dysphagia; the consequences of brain damage; ultrasound.

\section{Введение}

У пациентов, перенесших тяжелую ЧМТ, инсульты и хирургическое лечение опухолей головного мозга, находящихся в длительном хроническом критическом состоянии, имеется недостаточность питания вследствие различных органических и функциональных причин, в том числе нарушения глотания. Стационарные сроки лечения при этом длительные и иногда превышают несколько лет, и все это время пациенты лишены возможности самостоятельного питания естественным путем. В связи с этим у данной категории пациентов возникает вопрос обеспечения пути энтерального питания. Кроме того, у части пациентов и после выписки сохраняются затруднения в обеспечении перорального питания, в связи с чем метод энтерального питания должен быть максимально доступным и безопасным в обслуживании [3, 7]. В медицинской практике на данный момент существует два основных способа: назогастральный зонд и гастростома.

Учитывая выбранную группу пациентов, речь идет о длительном (несколько месяцев, а иногда и лет) обеспечении пути энтерального питания. Длительно функционирующий назогастральный зонд доставляет физический и психологический дискомфорт, довольно часто требует переустановки, вызывает эрозивные дефекты слизистой в местах контакта в пищеводе и желудке и к тому же мешает проведению ряда реабилитационных мероприятий, направленных на восстановление функции глотания (занятия с логопедом, массаж, физиотерапевтические процедуры, манипуляции на ЛОР-органах) $[14,17]$. Поэтому, мы считаем данный вариант обеспечения энтерального питания не совсем подходящим для данной 
категории пациентов, следовательно, наиболее оптимальным способом остается гастростомия.

По способу наложения гастростомию можно разделить на [16]:

1. Классическую (открытую) гастростомию по Витцелю, по Кадеру и др.

2. Лапароскопическую гастростомию.

3. Чрескожную эндоскопическую гастростомию (ЧЭГ).

Первые два способа являются полноценными оперативными вмешательствами с соответствующим риском, и требуют специального оснащения. Пациенты выбранной группы имеют крайне высокий операционный риск и, следовательно, данные способы для них имеют ряд ограничений [18].

ЧЭГ является «золотым стандартом» для прямого доступа в желудок во всем мире благодаря ряду преимуществ, таких как: возможность выполнения у пациентов с высоким операционным риском, возможность проведения в условиях отделении реанимации и эндоскопического кабинета, минимальная седация (нет необходимости в общем наркозе), небольшая длительность операции (15-30 мин). Помимо этого, уход за гастростомой, наложенной эндоскопическим методом, не требует никаких дополнительных мероприятий, прост и безопасен для больного, отсутствует опасность самопроизвольного зарастания свища, так как он выстлан на всем протяжении слизистой оболочкой желудка $[1,2,15]$. И, как следствие, является методом выбора для нашей категории пациентов. Так же стоит отметить, что ЧЭГ требует отмены реабилитационных мероприятий только в день проведения вмешательства и позволяет возобновить их в полном объеме уже на следующий день.

Однако при выполнении ЧЭГ также имеются ограничения.

Выделяют абсолютные и относительные противопоказания к ЧЭГ $[3,7,8]$ :

1. Абсолютные противопоказания:

- фарингеальная или эзофагеальная непроходимость, не позволяющая проводить гастроскопию;

- непроходимость желудка или кишечника, не позволяющая применить энтеральное питание;

- противопоказания для энтерального питания (анурия, острый тяжелый панкреатит);

- когулопатия (риск значительной кровопотери);

- выраженный асцит;

- терминальные состояния. 
2. Относительные противопоказания:

- осложненные язвы желудка и двенадцатиперстной кишки;

- субтотальная резекция желудка;

- вентральные грыжи;

- выраженная портальная гипертензия (выраженная гепатомегалия, спленомегалия);

- ожирение высокой степени;

- перитонеальный диализ.

Однако, как и при любой манипуляции, при выполнении ЧЭГ существуют риск интраоперационных осложнений, например, ранение печени, перфорация кишки. В среднем по данным литературы в 4,0-5,0\% случаев возникают осложнения, требующие дополнительного хирургического вмешательства.

Осложнения, которые возникают во время и после гастростомии 4,5$5,5 \%[3,6]$ :

1. Интраоперационные:

- кровотечение;

- перфорация;

- аспирация.

2. Послеоперационные:

- гастро-колоно-кожные свищи или толстокишечная непроходимость;

- перитонит, развивающиеся при случайной пункции ободочной кишки;

- перистомальные инфекции;

- развитие травматических эрозий или язв на противоположной от гастростомы стенке и под диском внутренней фиксации.

Осложнения, развивающиеся вследствие неправильного ухода за стомой $(4,0 \%)[3,6]$ :

- непроходимость гастростомической трубки, обычно вследствие введения густой смеси или лекарственных веществ;

- «бампер»-синдром - разрастание слизистой оболочки над диском для внутренней фиксации с закупоркой внутреннего отверстия зонда.

На настоящий момент существует 2 основных методики проведения ЧЭГ $[10,11,12]$ : «на себя» (Pull - техника Gauderer-Ponsky) и «от себя» (Push - техника Sacks-Vine). В ряде случаев применяется методика «интродуктор» (техника Russell) с использованием проводника и ряда расширителей для увеличения размера гастростомического свища, при которой возможная постановка более толстой трубки (катетер Фолея). Наиболее распространенным является метод «на себя». На наш взгляд данная ме- 
тодика является более удачной, чем «от себя», т.к. по технике Sacks-Vine дополнительно делается 3 прокола иглой, пексия стенки желудка к брюшной стенке и установка гастростомы извне. Это ведет к дополнительным интраоперационным рискам (перфорация органов, вероятность разрыва стенки желудка в местах фиксации во время установки гастростомы и пр.). При использовании метода Pull стенка желудка подтягивается изнутри к передней брюшной стенке и фиксируется непосредственно замком гастростомы без дополнительного натяжения. У нашей категории пациентов не было каких-либо противопоказаний к методике «на себя» (стенозы пищевода, препятствующие проведение эндоскопа), поэтому нами использовалась техника Gauderer-Ponsky.

В найденной нами литературе данных по использованию УЗ-навигации не обнаружено, хотя на наш взгляд подключение данного метода может повысить безопасность ЧЭГ.

\section{Цель настоящего исследования}

Целью настоящего исследования является анализ эффективности УЗ-навигации при ЧЭГ для повышения безопасности метода обеспечения пути доставки энтерального питания.

\section{Характеристика клинического материала, методы}

В ФНКЦ РР ЧЭГ была выполнена 115 пациентам, находящимся в длительном критическом состоянии, обусловленном органическим поражением головного мозга и имеющим стойкие нарушения глотания: 65 мужчин (57\%), 50 женщин (43\%). Возраст пациентов варьировал от 22 до 80 лет (средний возраст - 43 года).

Использовался набор для чрескожной эндоскопической гастростомии «Freka PEG» фирмы «Fresenius Kabi» (диаметр трубки - 20 Fr). Выполнялась методика «на себя» (pull) по Gauderer-Ponsky [5, 9]. Для УЗИ-навигации использовался аппарат DC-8 с принадлежностями фирмы Mindray c помощью линейного мультичастотного датчика высокой плотности L146WE, диапазон частот 4.8-10.6, 5.4-11.6, 6.6-13.5 МГц, 256 элементов, реальная апертура 56 мм или конвексного датчика C7-3E, диапазон частот 2.6-4.8, 3.6-6.4, 3.6-7.2 МГц.

Наложение гастростомы выполнялось под внутривенной анестезией двумя специалистами. Первым этапом была проведена видеоэзофагогастродуоденоскопия для исключения эрозивно-язвенных поражений желудка и луковицы двенадцатиперстной кишки. Далее при осмотре желудка 
проводилась диафаноскопия передней брюшной стенки, в результате которой выявлялась и отмечалась точка на передней брюшной стенке, соответствующая месту непосредственного прилежания передней стенки желудка. Далее проводилась пальпация передней брюшной стенки в отмеченной точке под контролем эндоскопа, при этом в желудке определяли чётко выраженное вдавление, образуемое пальцем. Затем проводилось УЗИ отмеченной области для исключения попадания других органов брюшной полости по ходу пункционной иглы. Передняя брюшная стенка обрабатывалась антисептическими растворами и обкладывалась стерильным материалом. Под местной анастезией (10 мл 0,25\% раствора новокаина) приступали к следующему этапу: производили разрез кожи длиной 1,0 см. Далее под контролем эндоскопа производили пункцию передней брюшной стенки и передней стенки желудка пункционной иглой из набора. Через пункционную иглу проводили нить в желудок, в дальнейшем захватывали ее эндоскопической петлей и выводили вместе с эндоскопом наружу через рот. К нити фиксировали гастростомическую трубку из набора, имеющую на одном конце диск для внутренней фиксации. Далее производили тракцию за нить со стороны передней брюшной стенки, и через пункционное отверстие желудка на переднюю брюшную стенку выводили гастростомическую трубку. Затем проводилась контрольная гастроскопия, оценивалось расположение и прилегание внутренней фиксирующей пластины, также исключалось кровотечение. Следующим этапом на гастростомическую трубку накладывали наружную фиксирующую пластину, клапан гастростомы и коннекторы. В течение первых суток всем пациентам выполнялась обзорная рентгенография брюшной полости для исключения пневмоперитонеума.

В наблюдаемой нами группе после проведения ЧЭГ начало питания пациентов осуществлялось по следующей схеме $[3,4,13]$ :

1. в 1-е сутки после установки гастростомы рекомендовано вводить глюкозо-электролитную смесь в объёме 500 мл, капельно со скоростью 100 мл/ч (например, Регидрон);

2. На следующие сутки объём глюкозо-электролитной смеси увеличивается до 1000 мл и начинает вводиться питание в объёме 500 мл, со скоростью 60-70 мл в час;

3. На 3 сутки пациенту вводят питание и глюкозоэлектролитную смесь в полном объёме в виде болюсов (с помощью шприца Жане).

Всем пациентам в группе проводилась периоперационная антибиотикопрофилактика - цефазолин 1,0 в/в за 30-40 мин до операции. 


\section{Результаты}

Послеоперационное осложнение наблюдалось всего в 2 случаях (1,7\%): пневмоперитонеум, выявленный посредством рентгенографии, при этом какие-либо клинические проявления отсутствовали. Обоим пациентам был назначен курс антибактериальной терапии, на 7-10-е сутки наблюдалось полное разрешение пневмоперитонеума, дополнительные хирургические вмешательства не потребовались. По данным мировой литературы осложнения возникают в 4,5-5,5\% случаев $[3,6,19]$.

Стоит отметить, что добавление в методику УЗИ области, определенной по диафаноскопии, оправдало себя в 15 -ти случаях $(13,0 \%)$ : из них с частотой $73,0 \%$ в определенной для пункции точки выявлен край печени, и с частотой $27,0 \%$ выявлена поперечно-ободочная кишка. После чего была определена другая точка для пункции и удалось избежать таких осложнений, как повреждение печени и перфорация кишки. По данным литературы перитонит, развивающийся при случайной пункции ободочной кишки, является одним из частых послеоперационных осложнений ЧЭГ $[3,6,20]$.

Замена гастростомической трубки из-за ее дисфункции была выполнена у 23-х $(20,0 \%)$ и из-за выпадения гастростомы у 8-ми $(7,0 \%)$ пациентов. В 2-х случаях проводилась замена гастростомической трубки, установленной «открытым» способом, при этом выявились технические сложности, обусловленные чрезмерной фиксацией трубки в канале гастростомы. В одном случае замена произведена из-за миграции внутреннего конца трубки с манжетой в двенадцатиперстную кишку и развитием у пациента реактивного панкреатита. После замены гастростомической трубки и на фоне консервативной терапии явления панкреатита купировались на 3-е сутки.

Необходимо отметить, что гастростома была удалена 15 пациентам $(13,0 \%)$ вследствие полного восстановления функции глотания. Закрытие гастростомического свища у всех пациентов наблюдалось в первую неделю.

Выполнение обзорной рентгенографии брюшной полости, по нашему мнению, так же является необходимым в 100\% случаев, так как позволяет выявить пневмоперитонеум и своевременно принять соответствующие меры и, как следствие, избежать более тяжелых осложнений.

\section{Выводы}

1. ЧЭГ по методике «pull» с добавлением УЗ-навигации является адекватным способом обеспечения пути энтерального питания у пациентов в 
длительном (хроническом) критическом состоянии, так как является малоинвазивной, технически простой и непродолжительной манипуляцией. ЧЭГ позволяет приступать к более раннему началу энтерального питания и не требует повторных операций для закрытия гастростомы. После выполнения ЧЭГ реабилитационные мероприятия могут быть возобновлены уже на следующий день и в полном объеме. Кроме того, ЧЭГ можно проводить в палате реанимации и эндоскопическом кабинете (при условии соблюдения всех правил антисептики).

2. Проведение УЗ-навигации области, определенной диафаноскопией, непосредственно перед пункцией желудка, особенно у пациентов с гепатомегалией, избыточной массой тела и у пациентов, имеющих в анамнезе оперативные вмешательства на брюшной полости, позволило избежать послеоперационных тяжелых осложнений в 13,0\% случаев, что доказывает целесообразность применения исследования, как часть ЧЭГ.

3. Выполняя ЧЭГ под УЗ-навигацией, удалось снизить число осложнений.

\section{Список литературы}

1. Балалыкин А.С., Козин С.М., Саввин В.Ю. и др. Чрескожная эндоскопическая гастростомия // Эндоскоп. хир., 2007. № 1. С. 115-116.

2. Волков О.И. Чрескожная эндоскопическая гастростомия.// Тихоокеанский медицинский журнал. г. Владивосток, 2004. № 1. С. 30-35.

3. Кузьмин-Крутецкий М.И., Сафоев М.И. Чрескожная эндоскопическая гастростомия в условиях многопрофильного стационара // Материалы конференции «Доброкачественные заболевания желудка у детей и взрослых». г. Санкт-Петербург, 2016, тезисы.

4. Иванов Ю.В., Сазонов Д.В., Таймаскина М.Т., Панченков Д.Н. Чрескожная эндоскопическая гастростомия: возможности, особенности, эффективность // Доктор.ру. г. Москва, 2015 С. 60-65.

5. Gauderer M.W. Percutaneous endoscopic gastrostomy -20 years later: a historical perspective // J. Pediatr. Surg. 2001. Vol. 36. p. 217-219.

6. Larson D.E., Burton D.D., Schroeder K.W., DiMagno E.P. Percutaneous endoscopic gastrostomy. Indications, success, complications, and mortality in 314 consecutive patients // Gastroenterology, 1987. Vol. 93. p. 48-52.

7. Marks J.M., Ponsky J.L. Access routes for enteral nutrition // Gastroenterologist. 1995. Vol. 3. p. 130-140.

8. Stellato T.A. Expanded applications of percutaneous endoscopic gastrostomy // Gastrointest Clin N Am. 1992. Vol. 2, pp. 249-257. 
9. Gauderer M.W.L., Ponsky J.L., Izant R.J. Gastrostomy without laparotomy: a percutaneous endoscopic technique // J. Pediatr. Surg. 1980. Vol. 15. P. 872.

10. Currie B.M., Getrajdman G.I., Covey A.M., Alago W.Jr., Erinjeri J.P., Maybody M., Boas F.E. Push versus pull gastrostomy in cancer patients: A single center retrospective analysis of complications and technical success rates // Diagn Interv Imaging. 2018, Apr 28.

11. Bergstrom L.R., Santhi Swaroop V., Zinsmeister A.R. Percutaneous endoscopic gastrostomy in patients with dementia // The American Journal of Gastroenterology. 2001. V. 96. № 1. P. 267.

12. Taylor A.L., Carroll T.A., Jakubowski J., O’Reilly G. Percutaneous endoscopic gastrostomy in patients with ventriculoperitoneal shunts // British Journal of Surgery. 2001. V. 88. № 5. P. 724-727.

13. Balihar K., Janská E., Zdrhová L., Kotyza J., Hejda V., Koželuhová J. Percutaneous endoscopic gastrostomy: analysis of practice at the endoscopic center of tertiary medical care // Vnitr Lek. Summer 2016;62(6):435-41.

14. Козин С.М., Вахотин А.Ю., Добродеев С.А., Балалыкин А.С. Чрескожная эндоскопическая гастростомия для длительного проведения энтерального питания // Вестник хирургической гастроэнтерологии. 2009. № 1. C. $26-33$.

15. Струков Е.Ю., Белевич В.Л., Щеголев А.В., Курыгин А.А. Чрескожная эндоскопическая гастростомия для длительного искусственнного питания тяжелобольных и пострадавших // Вестник хирургии им. И.И. Грекова. 2014. T. 173. № 4. C. 31-34.

16. Емельянов С.И., Новиков Г.А., Богданов Д.Ю., Матвеев Н.Л., Панченков Д.Н., Курганов И.А., Нечунаев А.А., Иванов Ю.В., Рудой С.В., Астахов Д.А., Соловьев Н.А., Введенская Е.С., Вайсман М.А., Подкопаев Д.В. Минимально инвазивные хирургические технологии в паллиативной медицинской помощи // Паллиативная медицина и реабилитация. 2017. № 3. С. 37-56.

17. Сорокин Ю.Н. Нарушения глотания при инсультах. 3. Особенности ведения пациента с дисфагией // Медицина неотложных состояний. 2015. № 3 (66). C. 144-149.

18. Хорошилов И.Е. Стандарты питания у реанимационных больных // Современные проблемы и инновационные технологии в анестезиологии и реаниматологии. Юбилейная научно-образовательная конференция: тезисы конференции. 2017. С. 60-61

19. Zhang L., Sanders L., Fraser R.J. Nutritional support teams increase percutaneous endoscopic gastrostomy uptake in motor neuron disease // World J Gastroenterol. 2012. Nov 28. 
20. Richter-Schrag H.J., Richter S., Ruthmann O., Olschewski M., Hopt U.T., Fischer A. Risk factors and complications following percutaneous endoscopic gastrostomy: a case series of 1041 patients // Can J Gastroenterol. 2011 Apr;25(4):201-6.

\section{Referenses}

1. Balalykin A.S., Kozin S.M., Savvin V.Yu. et al. Chreskozhnaya ehndoskopicheskaya gastrostomiya [Percutaneous endoscopic gastrostomy]. Ehndoskop. hir., 2007. No 1, pp. 115-116.

2. Volkov O.I. Chreskozhnaya ehndoskopicheskaya gastrostomiya [Percutaneous endoscopic gastrostomy]. Tihookeanskiu medicinskii zhurnal. Vladivostok, 2004. No 1, pp. 30-35.

3. Kuz'min-Kruteckiĩ M.I., Safoev M.I. Chreskozhnaya ehndoskopicheskaya gastrostomiya $v$ usloviyah mnogoprofil'nogo stacionara [Percutaneous endoscopic gastrostomy in conditions of multi-profile hospital]. Materials of conference «Dobrokachestvennye zabolevaniya zheludka u detej i vzroslyh». Sankt-Peterburg, 2016.

4. Ivanov Yu.V., Sazonov D.V., Tajmaskina M.T., Panchenkov D.N. Chreskozhnaya ehndoskopicheskaya gastrostomiya: vozmozhnosti, osobennosti, ehffektivnost' [Percutaneous endoscopic gastrostomy: possibilities, features, efficiency]. Doktor.ru. Moscow, 2015, pp. 60-65.

5. Gauderer M.W. Percutaneous endoscopic gastrostomy -20 years later: a historical perspective. J. Pediatr. Surg. 2001. Vol. 36, pp. 217-219.

6. Larson D.E., Burton D.D., Schroeder K.W., DiMagno E.P. Percutaneous endoscopic gastrostomy. Indications, success, complications, and mortality in 314 consecutive patients. Gastroenterology, 1987. Vol. 93, pp. 48-52.

7. Marks J.M., Ponsky J.L. Access routes for enteral nutrition. Gastroenterologist. 1995. Vol. 3, pp. 130-140.

8. Stellato T.A. Expanded applications of percutaneous endoscopic gastrostomy. Gastrointest Clin N Am. 1992. Vol. 2, pp. 249-257.

9. Gauderer M.W.L., Ponsky J.L., Izant R.J. Gastrostomy without laparotomy: a percutaneous endoscopic technique. J. Pediatr. Surg. 1980. Vol. 15. P. 872.

10. Currie B.M., Getrajdman G.I., Covey A.M., Alago W.Jr., Erinjeri J.P., Maybody M., Boas F.E. Push versus pull gastrostomy in cancer patients: A single center retrospective analysis of complications and technical success rates. $D i$ agn Interv Imaging. 2018. Apr 28.

11. Bergstrom L.R., Santhi Swaroop V., Zinsmeister A.R. Percutaneous endoscopic gastrostomy in patients with dementia. The American Journal of Gastroenterology. 2001. V. 96. № 1. P. 267. 
12. Taylor A.L., Carroll T.A., Jakubowski J., O’Reilly G. Percutaneous endoscopic gastrostomy in patients with ventriculoperitoneal shunts. British Journal of Surgery. 2001. V. 88. № 5, pp. 724-727.

13. Balihar K., Janská E., Zdrhová L., Kotyza J., Hejda V., Koželuhová J. Percutaneous endoscopic gastrostomy: analysis of practice at the endoscopic center of tertiary medical care. Vnitr Lek. Summer 2016;62(6):435-41.

14. Kozin S.M., Vahotin A.Yu., Dobrodeev S.A., Balalykin A.S. Chreskozhnaya ehndoskopicheskaya gastrostomiya dlya dlitel'nogo provedeniya ehnteral'nogo pitaniya [Percutaneous endoscopic gastrostomy for long-term enteral nutrition]. Vestnik hirurgicheskoj gastroehnterologii. 2009. № 1, pp. 26-33.

15. Strukov E.Yu., Belevich V.L., Shchegolev A.V., Kurygin A.A. Chreskozhnaya ehndoskopicheskaya gastrostomiya dlya dlitel'nogo iskusstvennnogo pitaniya tyazhelobol'nyh i postradavshih [Percutaneous endoscopic gastrostomy for long-term artificial feeding of seriously ill and injured patients]. Vestnik hirurgii im. I.I. Grekova. 2014. vol. 173. № 4, pp. 31-34.

16. Emel'yanov S.I., Novikov G.A., Bogdanov D.Yu., Matveev N.L., Panchenkov D.N., Kurganov I.A., Nechunaev A.A., Ivanov Yu.V., Rudoj S.V., Astahov D.A., Solov'ev N.A., Vvedenskaya E.S., Vajsman M.A., Podkopaev D.V. Minimal'no invazivnye hirurgicheskie tekhnologii $\mathrm{v}$ palliativnoj medicinskoj pomoshchi [Minimally invasive surgical technologies in palliative care]. Palliativnaya medicina i reabilitaciya. 2017. № 3, pp. 37-56.

17. Sorokin Yu.N. Narusheniya glotaniya pri insul'tah. 3. Osobennosti vedeniya pacienta s disfagiej [Of swallowing disorders in stroke. 3. Peculiarities of management of patients with dysphagia]. Medicina neotlozhnyh sostoyanij. 2015. № 3 (66), pp. 144-149.

18. Horoshilov I.E. Standarty pitaniya u reanimacionnyh bol'nyh [Standards of nutrition in intensive care patients]. Sovremennye problemy $i$ innovacionnye tekhnologii v anesteziologii i reanimatologii [Jubilee scientific and educational conference]: conference abstracts. 2017, pp. 60-61.

19. Zhang L., Sanders L., Fraser R.J. Nutritional support teams increase percutaneous endoscopic gastrostomy uptake in motor neuron disease. World J Gastroenterol. 2012 Nov 28.

20. Richter-Schrag H.J., Richter S., Ruthmann O., Olschewski M., Hopt U.T., Fischer A. Risk factors and complications following percutaneous endoscopic gastrostomy: a case series of 1041 patients. Can J Gastroenterol. 2011 Apr;25(4):201-6.

\section{ДАННЫЕ ОБ АВТОРАХ}

Яковлева Александра Витальевна, младший научный сотрудник лаборатории нутригеномики и нутригенетики 
Шайбак Александр Анатольевич, врач-эндосокопист

Скворцов Артем Евгеньевич, заведующий отделением функциональной диагностики

Яковлев Алексей Александрович, заведующий хирургическим отделением с дневным стационаром

Крылов Кирилл Юрьевич, к.м.н., заведующий лабораторией нутригеномики и нутригенетики

Петрова Марина Владимировна, д.м.н., профессор, заместитель директора по научно-клинической деятельности - руководитель НИИ Реабилитологии

Щелкунова Инесса Геннадиевна, к.м.н., доцент, Заместитель директора по развитию внебюджетной деятельности и стратегическому менеджменту

Гречко Андрей Вячеславович, д.м.н., профессор, профессор РАН, директор ФНКЦ РР

Федеральное государственное бюджетное научное учреждение "Федеральный научно-клинический центр реаниматологии и реабилитологии» (ФНКЦ РР)

ул. Петровка, 25, стр.2, г. Москва, 107031, Российская Федерация mogvv2016@yandex.ru

DATA ABOUT THE AUTHORS

Yakovleva Alexandra Vitalievna, Junior researcher of the laboratory of nutrigenomic and nutrigenetics

Sibak Alexander Anatolevich, Endoscopist

Skvortsov Artem Evgenievich, Head of functional Diagnostics Department

Yakovlev Alexey Aleksandrovich, Head of the Surgical Department and Daycare facility

Krylov Kirill Yurievich, PhD, Head of the laboratory of nutrigenomics and nutrigenetics 
Petrova Marina Vladimirovna, MD, Professor, Deputy Director for scientific and clinical activities-head of the research NII Rehabilitologii

Shchelkunova Inessa Gennadiyevna, $\mathrm{PhD}$, docent, Deputy Director for development of off-budget activities and strategic management

Grechko Andrey Vyacheslavovich, MD, Professor, Professor of the Russian Academy of Sciences, Director of Federal Research and Clinical Center of Intensive Care Medicine and Rehabilitology

Federal Research and Clinical Center of Intensive Care Medicine and Rehabilitology

25, p. 2, Petrovka Str., Moscow, 107031, Russian Federation mogvv2016@yandex.ru 